\title{
LINPRO: linear inverse problem library for data contaminated by statistical noise ${ }^{\text {th }}$
}

\author{
Piotr Magierski, Gabriel Wlazłowski \\ Faculty of Physics, Warsaw University of Technology, ulica Koszykowa 75, 00-662 \\ Warsaw, POLAND
}

\begin{abstract}
The library LINPRO which provides the solution to the linear inverse problem for data contaminated by a statistical noise is presented. The library makes use of two methods: Maximum Entropy Method and Singular Value Decomposition. As an example it has been applied to perform an analytic continuation of the imaginary time propagator obtained within the Quantum Monte Carlo method.

Keywords: Linear Inverse Problem, Maximum Entropy Method, Singular Value Decomposition
\end{abstract}

\section{Program Summary}

Title of the program: LINPRO v1.0

Catalogue number: ....

Program obtainable from: CPC Program Library, Queen's University of Belfast, N. Ireland (see application form in this issue)

Licensing provisions: GNU Lesser General Public Licence.

Distribution format: tar.gz

Programming language: $\mathrm{C}++$

Technical and API documentation: Yes, in HTML format

Computer: LINPRO library should compile on any computing system that has $\mathrm{C}++$ compiler.

\footnotetext{
This paper and its associated computer program are available via the Computer Physics Communications homepage on ScienceDirect
} 
Operating systems: LINUX or UNIX.

Tested with compilers: GNU Compiler g++, Intel Compiler icpc.

External libraries: OPT++: An Object-Oriented Nonlinear Optimization Library [8] (included into distribution).

No. of lines in distributed program, source files only: 8517.

Nature of problem: LINPRO library solves linear inverse problem with an arbitrary kernel and arbitrary external constraints imposed on the solution. Solution method: LINPRO library implements two complementary methods: Maximum Entropy Method and SVD method.

\section{Linear inverse problem}

\subsection{Formulation of the problem}

The inverse problem considered here is of the form:

$$
G(y)=\int_{-\infty}^{\infty} K(x, y) A(x) d x
$$

where $y \in(\alpha, \beta)$ and the kernel $K$ is a known, real function, sufficiently regular, although not necessarily smooth. The function $G$ is known, and is represented by a finite number $N_{\tau}$ of values at a given set of points: $\left(y_{1}, y_{2}, \ldots, y_{N_{\tau}}\right)$. The values $G\left(y_{i}\right)=G_{i}$ and $\vec{G}=\left(G_{1}, G_{2}, \ldots, G_{N_{\tau}}\right)^{T}$ will be called the data and the data vector, respectively. These values are assumed to be in addition affected by a noise of statistical origin and has to be treated merely as approximations of the true values. The unknown function $A$ will be called the object, irrespective to its physical nature. The object is assumed to be nonzero only within a finite interval $(a, b)$, although $a$ and $b$ are in general unknown. Moreover $A$ may be a subject of additional constraints of the form:

$$
\int_{-\infty}^{\infty} g_{i}(x) A(x) d x=c_{i}, \quad i=1,2, \ldots, L
$$

and

$$
A\left(x_{j}\right) \in\left[l_{j}, u_{j}\right], \quad j=1,2, \ldots, M,
$$

where functions $g_{i}$ and values $c_{i}$ are known, $l_{j}$ and $u_{j}$ indicate the lower and upper bound imposed on the object at some point $x_{j}$. 


\subsection{Normal solution}

Since the function $G$ is known for the finite set of argument values the linear inverse problem (11) in practice reduces to its discretized counterpart:

$$
G_{i}=\int_{-\infty}^{\infty} K\left(x, y_{i}\right) A(x) d x=\int_{-\infty}^{\infty} K_{i}^{*}(x) A(x) d x=\left(K_{i}, A\right),
$$

where $(\cdot, \cdot)$ denotes the inner product. The object $A$ can be treated as an element of $N$-dimensional Hilbert space $\mathcal{A}_{N}$ (in general $N=\infty$ ). Note that due to discretization the kernel functions $K_{i}(x)$ span only $M$-dimensional subspace $\mathcal{A}_{M}\left(M \leq N_{\tau}\right)$ of the space $\mathcal{A}_{N}$. It makes the inverse problem illposed, as there exists an infinite class of solutions satisfying Eq. (4). Indeed, let us expand $K_{i}$ and $A$ in an orthonormal basis $\left\{u_{k}\right\}_{k=1}^{N}$ in $\mathcal{A}_{N}$ :

$$
\begin{aligned}
K_{i}(x) & =\sum_{k=1}^{M} f_{i k} u_{k}(x), \\
A(x) & =\sum_{k=1}^{N} a_{k} u_{k}(x)=\sum_{k=1}^{M} a_{k} u_{k}(x)+\sum_{k=M+1}^{N} a_{k} u_{k}(x) \\
& =A_{P}(x)+A_{\perp}(x),
\end{aligned}
$$

where $A_{P} \in \mathcal{A}_{M}$ represents the projection of $A$ onto the $M$-dimensional subspace of $\mathcal{A}_{N}$ and $A_{\perp}$ is the remaining part, orthogonal to $A_{P}:\left(A_{P}, A_{\perp}\right)=$ 0 . Substituting the above expansions to Eq. (44) one gets

$$
G_{i}=\sum_{k=1}^{M} \sum_{l=1}^{N} f_{i k}^{*} a_{l}\left(u_{k}, u_{l}\right)=\sum_{k=1}^{M} f_{i k}^{*} a_{k},
$$

where we have used the property $\left(u_{k}, u_{l}\right)=\delta_{k l}$. The last equality shows that $G_{i}$ is independent of $A_{\perp}$, since $G_{i}=\left(K_{i}, A\right)=\left(K_{i}, A_{P}\right)$. It implies that the data vector $\vec{G}$ allows only for the reconstruction of $A_{P}$. The solution $A_{P}$ with the minimal norm is a unique element of the subspace $\mathcal{A}_{N}$ and is called the normal solution [1].

In the case of data contaminated by a statistical noise the solution of the problem is also affected by uncertainties. Below we present two strategies which allow us to deal with such problems:

1. the singular system analysis which uses the Singular Value Decomposition (SVD) to determine $A_{P}$ and subsequently decrease uncertainties of the normal solution by incorporating constraints imposed on $A$, 
2. the Maximum Entropy Method (MEM), which finds the most probable solution, under the condition that data represent random numbers normally distributed around the true values, and that certain objects $A$ are more probable than the others (so called a priori information about the object $A$ ).

\section{Singular system analysis}

\subsection{SVD Method}

The normal solution $A_{P}$ can be determined using the singular value decomposition of the integral kernel in Eq. (4) [1, 2, 3, 4]. Let us rewrite it in the form:

$$
\vec{G}=\mathcal{K} A .
$$

The kernel functions $K_{i}(x)$ span $M$-dimensional subspace $\mathcal{A}_{M}$ and therefore $\vec{G}$ has only $M$ independent elements [1]. Thus $\vec{G}$ is an element of $M$ dimensional vector space $\mathcal{G}_{M}^{N_{\tau}}$. $\mathcal{K}$ is an integral operator which transforms an object from $\mathcal{A}_{N}$-space into a vector of the data space $\mathcal{G}_{M}^{N_{\tau}}$. The operator can be treated as a rectangular matrix of dimension $N_{\tau} \times N$. We can define also a conjugate operator $\mathcal{K}^{\dagger}$ which transforms vectors from $\mathcal{G}_{M}^{N_{\tau}}$-space into $\mathcal{A}_{N}$-space using the relation:

$$
(\mathcal{K} u, \vec{v})=\left(u, \mathcal{K}^{\dagger} \vec{v}\right)
$$

where $u \in \mathcal{A}_{N}, \vec{v} \in \mathcal{G}_{M}^{N_{\tau}}$ and the inner product in the data space is defined as follows:

$$
\left(\vec{v}, \overrightarrow{v^{\prime}}\right)=\sum_{i=1}^{N_{\tau}} v_{i} v_{i}^{\prime},
$$

which is a useful definition in the case of uncorrelated data 1 . The conjugate operator $\mathcal{K}^{\dagger}$ can be treated as a rectangular matrix of dimension $N \times N_{\tau}$. Consequently the operator $\mathcal{K K}^{\dagger}$ is represented by a square matrix of dimension $N_{\tau} \times N_{\tau}$. Matrix elements of the operator $\mathcal{K} \mathcal{K}^{\dagger}$ are simply given by

$$
\left(\mathcal{K K}^{\dagger}\right)_{i j}=\left(K_{i}, K_{j}\right)
$$

\footnotetext{
${ }^{1}$ In the case of correlated data it is more appropriate to define the inner product as $\left(\vec{v}, \overrightarrow{v^{\prime}}\right)=\sum_{i, j=1}^{N_{\tau}} v_{i} W_{i j} v_{j}$, where the matrix $W$ is the inverse of the covariance matrix.
} 
Performing the diagonalization of the matrix $\mathcal{K K}^{\dagger}$ enables to determine the dimension of the subspace spanned by the kernel functions $K_{i}$. Indeed, the operator $\mathcal{K} \mathcal{K}^{\dagger}$ has $M$ positive eigenvalues $\left\{\lambda_{i}^{2}\right\}_{i=1}^{M}$, where $M$ is the rank of the operator $\mathcal{K} \mathcal{K}^{\dagger}$. Corresponding eigenvectors $\left\{\vec{v}_{i}\right\}_{i=1}^{M}$ form a basis in the data space. Conjugate operator $\mathcal{K}^{\dagger} \mathcal{K}$, which acts in the object space $\mathcal{A}_{N}$, among its eigenvalues has the same positive eigenvalues as the operator $\mathcal{K K}^{\dagger}$ and its eigenfunctions $\left\{u_{i}\right\}_{i=1}^{M}$ form the basis of $\mathcal{A}_{M}$-space. The eigenvalues $\left\{\lambda_{i}^{2}\right\}$, the eigenvectors $\left\{\vec{v}_{i}\right\}$ and the eigenfunctions $\left\{u_{i}\right\}$ form a singular system of the operator $\mathcal{K}$ satisfying the shifted eigenvalue problem:

$$
\mathcal{K} u_{i}=\lambda_{i} \vec{v}_{i}, \quad \mathcal{K}^{\dagger} \vec{v}_{i}=\lambda_{i} u_{i}
$$

The numbers $\lambda_{i}$ are singular values, and $u_{i}, \vec{v}_{i}$ are singular functions and singular vectors, respectively. The definition of the conjugate operator $\mathcal{K}^{\dagger}$ and equations (12) allow to express the singular functions in the form:

$$
u_{i}(x)=\frac{1}{\lambda_{i}} \sum_{k=1}^{N_{\tau}} K_{k}(x)\left(\vec{v}_{i}\right)_{k}
$$

where $\left(\vec{v}_{i}\right)_{k}$ denotes $\mathrm{k}$-th element of vector $\left(\vec{v}_{i}\right)$.

The singular system forms a suitable basis for expansion of the unknown object $A_{P}[1]$ :

$$
A_{P}(x)=\sum_{i=1}^{M} b_{i} u_{i}(x),
$$

where the expansion coefficients are given by

$$
b_{i}=\frac{\left(\vec{v}_{i}, \vec{G}\right)}{\lambda_{i}} \text {. }
$$

\subsection{Data with noise}

The solution given by Eq. (14) can be used only in the case of noiseless data. In the case when data vector $\vec{G}$ is known with some uncertainty $\Delta \vec{G}$ the above algorithm becomes numerically ill-conditioned [1, 2, 3]. Note that the singular values $\left\{\lambda_{i}\right\}$, the singular vectors $\left\{\vec{v}_{i}\right\}$ and therefore the singular functions $\left\{u_{i}\right\}$ are known exactly since they are fully determined by the kernel functions $K_{i}$. Errors $\Delta \vec{G}$ affect only the expansion coefficients, which will be the subject to some uncertainty $\Delta b_{i}=\left(\vec{v}_{i}, \Delta \vec{G}\right) / \lambda_{i}$. To perceive the origin 
of numerical instabilities let us arrange the set of singular values $\left\{\lambda_{i}\right\}_{i=1}^{M}$ in descending order: $\lambda_{1} \geqslant \lambda_{2} \geqslant \ldots \geqslant \lambda_{M}$. Clearly, with a decreasing singular value the contribution of the statistical noise to $A_{P}$ is amplified:

$$
\lambda_{i} \rightarrow 0 \Rightarrow \Delta b_{i}=\frac{\left(\vec{v}_{i}, \Delta \vec{G}\right)}{\lambda_{i}} \rightarrow \infty
$$

Practically it means that in the object space $\mathcal{A}_{M}$ there exist "directions" which are invisible for the SVD method, namely, the expansion coefficients cannot be determined with sufficient accuracy starting from some $i$ index. Simultaneously the corresponding singular functions $u_{i}$ become rapidly oscillating with an increasing index $i$ (number of nodes of $i$-th singular function is $i-1$ ) [2]. The functions associated with smaller values of $\lambda_{i}$ are responsible for reconstructing more subtle details of the solution. Since large uncertainties of coefficients in general yield to strong fluctuations of the solution, one of the standard methods is to remove all such strongly fluctuating terms and include only those for which $b_{i}$ are determined with satisfactory accuracy:

$$
A_{P_{\text {cut }}}(x)=\sum_{i=1}^{M_{\text {cut }}} b_{i} u_{i}(x)
$$

This approach leads to the so called truncated SVD method (TSVD). In practice the truncation parameter is chosen in such a way to remove all terms for which the ratio (cut-off parameter) $\lambda_{i} / \lambda_{1}$ is smaller than $\frac{1}{N_{\tau}} \sum_{i=1}^{N_{\tau}} \frac{\Delta G_{i}}{\left|G_{i}\right|}$. It ensures that the solution $A_{P_{\text {cut }}}$ reproduces data $G_{i}$ within its error bars and prevents the inclusion of unjustified structures into the solution [2, 3].

\subsection{Incorporating a priori information}

The reconstruction quality of the SVD method decreases significantly if data are affected by even a relatively weak noise. It turns out however that the incorporation of a priori information can improve the reconstruction process [4, 5]. There are two types of the prior information: information concerning the support of the solution (interval where the solution is nonzero) and external constraints. The first type of information leads to the following modification of the original problem:

$$
G_{i}=\int_{-\infty}^{+\infty} K_{i}^{*}(x) A(x) d x \cong \int_{a}^{b} K_{i}^{*}(x) A(x) d x=\int_{-\infty}^{+\infty} K_{i}^{*}(x) S(x, a, b) A(x),
$$


where object $A$ is assumed to be non zero in the interval $(a, b) . S(x, a, b)$ denotes the support function defined as

$$
S(x, a, b)=\left\{\begin{array}{ll}
1, & \text { if } x \in(a, b) \\
0, & \text { if } x \notin(a, b)
\end{array},\right.
$$

which modifies the kernel functions for the SVD method. This modification has two major consequences. First, the singular values $\lambda_{i}$ decrease faster as the size $|b-a|$ of the support function gets smaller. It subsequently leads to smaller values of $M_{\text {cut }}$ and in general decreases the reconstruction ability of the method. Second, however, the singular functions $u_{i}$ become limited to the interval $x \in(a, b)$ and their zeros are spaced more closely. This implies that a smaller number of singular functions are needed to get the same accuracy of reconstruction as before. It turns out that the latter consequence dominates and a properly chosen support function increases reconstruction quality [5].

Within the SVD method it is also possible to generate the solution which satisfies integral constraints (2). This can be done using the fact that each solution of the form

$$
\tilde{A}_{P}\left(x,\left\{\tilde{b}_{i}\right\}\right)=\sum_{i=1}^{M} \tilde{b}_{i} u_{i}(x),
$$

where $\tilde{b}_{i} \in\left(b_{i}-\Delta b_{i}, b_{i}+\Delta b_{i}\right)$ reproduces the data $G_{i}$ within its error bars. Hence choosing an appropriate set of the expansion coefficients $\left\{\tilde{b}_{i}\right\}_{i=1}^{M}$ one can try to reproduce constraints (it is not always possible since the normal solution need not fulfill the same constraints as the true solution) [4]. In general, the expansion coefficients $\left\{\tilde{b}_{i}\right\}_{i=1}^{M}$ which agree with the constraints are not unique. To distinguish between various possibilities one can define the cost functional $\mathcal{C}\left[\tilde{A}_{P}\right]$, which has to be minimized to find the best set of coefficients. As a cost functional one can use $\chi^{2}$ statistics with a similar form like in the maximum entropy method (see next section). Another possibility is to choose the cost functional as the norm of the solution, $\mathcal{C}\left[\tilde{A}_{P}\right]=\left\|\tilde{A}_{P}\right\|=\sqrt{\left(A_{P}, A_{P}\right)}$. This choice is in agreement with the spirit of an SVD approach, where the normal solution is defined as the solution with the minimal norm. Summarizing, the problem of determining the unknown object satisfying external constraints has been reduced to the optimization problem:

$$
\tilde{A}_{P}(x)=\min _{\left\{\tilde{b}_{i}\right\}} \mathcal{C}\left[\tilde{A}_{P}\left(x,\left\{\tilde{b}_{i}\right\}\right)\right]
$$


with external constraints:

$$
\begin{aligned}
& \forall i=1,2, \ldots, M \quad: \quad b_{i}-\Delta b_{i} \leqslant \tilde{b}_{i} \leqslant b_{i}+\Delta b_{i}, \\
& \forall j=1,2, \ldots, L \quad: \quad \int_{-\infty}^{+\infty} g_{j}(x) \tilde{A}_{P}(x) d x=c_{j} .
\end{aligned}
$$

\section{Maximum Entropy Method}

\subsection{General considerations}

Let us distinguish between the exact values of the function $G$ which are unknown and fulfill Eq. (1) and their known approximations contained in a vector: $\overrightarrow{\tilde{G}}=\left(\tilde{G}_{1}, \tilde{G}_{2}, \ldots, \tilde{G}_{N_{\tau}}\right)^{T}$. These values can be treated as a particular realization of random variables, which are assumed to be uncorrelated 2 and have a normal distribution around the exact values $G_{i}$ with a variance $\sigma_{i}^{2}$. The probability of obtaining the particular realization $\overrightarrow{\tilde{G}}$ under the condition that the exact values are given by $\vec{G}$ reads

$$
p(\overrightarrow{\tilde{G}} \mid \vec{G}) \propto \exp \left(-\frac{1}{2} \sum_{i=1}^{N_{\tau}}\left(\frac{\tilde{G}_{i}-G_{i}}{\sigma_{i}}\right)^{2}\right),
$$

and the values $G_{i}$ depend on the function $A$ according to the relation (44). This equation is subsequently discretized in a chosen interval $(a, b)$ and becomes a linear transformation:

$$
G_{i}=\sum_{j=1}^{N} K_{i j} A_{j}
$$

where $K_{i j}=K\left(x_{j}, y_{i}\right) \Delta x$ is a rectangular matrix $N_{\tau} \times N, \Delta x=x_{j}-x_{j-1}$ and $A_{j}=A\left(x_{j}\right)$. Points $x_{j}$ are uniformly distributed over the interval $(a=$ $\left.x_{1}, b=x_{N}\right)$.

The estimator for the quantity $\vec{A}=\left(A_{1}, A_{2}, \ldots, A_{N}\right)^{T}$ is defined as the one which maximizes the conditional probability $p(\vec{A} \mid \tilde{G})$. This in turn can be expressed by (24) using Bayes' theorem:

$$
p(\vec{A} \mid \overrightarrow{\tilde{G}})=\frac{p(\overrightarrow{\tilde{G}} \mid \vec{G}) p(\vec{A})}{p(\overrightarrow{\tilde{G}})},
$$

\footnotetext{
${ }^{2}$ The extension of the method to the case of correlated data is straightforward, but require additional information in the form of covariance matrix.
} 
where on the rhs the dependence on $\vec{A}$ is included in $\vec{G}$ through the relation (25). The probability $p(\vec{A})$ is a priori probability and may contain additional information about $\vec{A}$ including constraints (2). The maximization of this probability (so called likelihood function) leads in practice to the condition:

$$
\frac{\partial}{\partial A_{j}} p(\vec{A} \mid \overrightarrow{\tilde{G}})=0, \quad \mathrm{j}=1, . ., \mathrm{N}
$$

In the case of $N \leq N_{\tau}$ and $p(\vec{A})=$ const the above condition reduces to the least square problem with the solution: $\vec{A}=\left(K^{T} K\right)^{-1} \vec{G}^{T} K$, where $\sigma_{i}=\sigma=$ const is assumed.

Here we are interested in the case when $N>N_{\tau}$ and an additional prior information is needed. It is specified through the entropy $S(\vec{A})$, where $p(\vec{A}) \propto$ $\exp (S(\vec{A}))$. The completely non-informative entropy is of the form:

$$
S(\vec{A})=-\alpha \sum_{i=1}^{N}\left(\frac{A_{i}}{\sum_{j=1}^{N} A_{j}}\right) \log \left(\frac{A_{i}}{\sum_{j=1}^{N} A_{j}}\right)
$$

where $\alpha>0$ is arbitrary. It favors the solution $\vec{A}=$ const. Usually we have additional information about the structure of $A$ which allows us to specify a model of $\vec{A}$. In such a case the relative entropy can be constructed:

$$
S(\vec{A} \mid \overrightarrow{\mathcal{M}})=-\alpha \sum_{i=1}^{N} \frac{A_{i} \log \frac{A_{i}}{\mathcal{M}_{i}}}{\sum_{j=1}^{N} A_{j}}
$$

where $\overrightarrow{\mathcal{M}}=\left(\mathcal{M}_{1}, \ldots, \mathcal{M}_{N}\right)^{T}, \mathcal{M}_{i}=\mathcal{M}\left(x_{i}\right)$ is an assumed model for $\vec{A}$ and $\sum_{i=1}^{N} A_{i}=\sum_{i=1}^{N} \mathcal{M}_{i}$. The model $\overrightarrow{\mathcal{M}}$ has to fulfill the constraints imposed on $\vec{A}$. In order to be able to construct the entropy in the above form, requires the assumption of nonnegativity of $A$. In order to avoid a complicated notation we assume also that both $\vec{A}$ and $\overrightarrow{\mathcal{M}}$ are normalized: $\sum_{i=1}^{N} A_{i}=\sum_{i=1}^{N} \mathcal{M}_{i}=1$. Clearly the entropy is maximized in the case when $\vec{A}=\overrightarrow{\mathcal{M}}$, although note that $S(\vec{A} \mid \overrightarrow{\mathcal{M}}) \neq S(\overrightarrow{\mathcal{M}} \mid \vec{A})$.

The prior information provides additional conditions for $\vec{A}$ and makes the maximization of the likelihood function a well defined process with a unique solution. Clearly now:

$$
p(\vec{A} \mid \tilde{G}) \propto \exp \left(-\frac{1}{2} \sum_{i=1}^{N_{\tau}}\left(\frac{\tilde{G}_{i}-G_{i}}{\sigma_{i}}\right)^{2}-\alpha \sum_{i=1}^{N} A_{i} \log \frac{A_{i}}{\mathcal{M}_{i}}\right),
$$


and the maximum entropy method leads to the maximization of the above function with respect to $\vec{A}[\underline{6}]$. Note that still one has a freedom of choosing the constant $\alpha>0$. It governs the relative importance of the two terms in the above expression and larger $\alpha$ favors the model over the data.

Another extension of the above formulation which will be considered in the next section admits the possibility of having a class of models $\overrightarrow{\mathcal{M}}(x ; \vec{f})$, where $\vec{f}$ is a set of parameters describing admissible degrees of freedom of the model and thus defining a set of admissible models.

\subsection{Method of solution}

The quantity which has to be minimized as a result of the MEM reads:

$$
F(\vec{A})=\frac{1}{2} \sum_{i=1}^{N_{\tau}}\left(\frac{\tilde{G}_{i}-G_{i}}{\sigma_{i}}\right)^{2}+\alpha \sum_{i=1}^{N} A_{i} \log \frac{A_{i}}{\mathcal{M}_{i}} .
$$

The task of minimizing the function of $N$ variables, where $N$ in practice may be of the order of $10^{2-4}$ is rather hard. Therefore we apply here the procedure described in Ref. [7] which replaces the minimization of the many-variable function by an iterative process of consecutive least square problems. Let us assume that $F\left(\vec{A}^{0}\right)=\min$ and $\vec{A}^{0}$ represents the solution of the problem. We expand $F$ around $\vec{A}^{0}$ up to the second order:

$$
\begin{aligned}
& F\left(\vec{A}^{0}+\delta \vec{A}\right)=F\left(\vec{A}^{0}, \vec{A}\right)= \\
= & \frac{1}{2} \sum_{i=1}^{N_{\tau}}\left(\frac{\tilde{G}_{i}-G_{i}}{\sigma_{i}}\right)^{2}+\alpha \sum_{i=1}^{N}\left(\frac{1}{2 A_{i}^{0}}\left(\gamma_{i}-A_{i}\right)^{2}+\omega_{i}\right)+O\left(|\delta \vec{A}|^{3}\right),
\end{aligned}
$$

where

$$
\begin{aligned}
\gamma_{i} & =A_{i}^{0}\left(1-\log \frac{A_{i}^{0}}{\mathcal{M}_{i}}\right) \\
\omega_{i} & =\mathcal{M}_{i}-A_{i}^{0}\left(1-\log \frac{A_{i}^{0}}{\mathcal{M}_{i}}+\frac{1}{2}\left(\log \frac{A_{i}^{0}}{\mathcal{M}_{i}}\right)^{2}\right), \\
\vec{A} & =\vec{A}^{0}+\delta \vec{A} .
\end{aligned}
$$

The above expansion implies the method of solving the problem. Namely, in the step $n$ we minimize $F\left(\vec{A}^{0(n)}, \vec{A}^{(n)}\right)$ with respect to $\vec{A}^{(n)}$ at fixed $\vec{A}^{0(n)}$. This is equivalent to the least square problem. Then we define a new $\vec{A}^{0(n+1)}=$ 
$\xi \vec{A}^{(n+1)}+(1-\xi) \vec{A}^{(n)}$, where $\xi \in(0,1)$. Such a procedure leads to a convergent solution providing $\xi$ is sufficiently small. As a starting condition one takes $\vec{A}^{(0)}=\vec{A}^{(1)}=\overrightarrow{\mathcal{M}}$.

The additional constraints (2) can be included by considering the modified function:

$$
G\left(\vec{A}^{0}, \vec{A}\right)=F\left(\vec{A}^{0}, \vec{A}\right)+\sum_{i=1}^{L} \theta_{i}\left(c_{i}-\sum_{j=1}^{N} g_{i, j} A_{j}\right)^{2},
$$

where $g_{i, j}=g_{i}\left(x_{j}\right) \Delta x$, and $\theta_{i}$ are positive parameters governing the "stiffness" of the constraints and thus responsible for the accuracy at which conditions (2) are fulfilled.

In the MEM approach we have improved the method of finding the solution by constructing a sequence of minimizations with a gradually refined model. In this case the model is of the form $\overrightarrow{\mathcal{M}}(x ; \vec{f})$ and thus represents a class of models defined by parameters $\vec{f}=\left(f_{1}, \ldots, f_{s}\right)$. At the end of each minimization process described above the result has been used to define a new model which maximize the overlap with respect to parameters $\vec{f}$.

$$
O(\vec{f})=\frac{\left(\sum_{i=1}^{N} A_{i} \mathcal{M}_{i}(\vec{f})\right)^{2}}{\sum_{i=1}^{N} A_{i}^{2} \sum_{i=1}^{N} \mathcal{M}_{i}^{2}(\vec{f})},
$$

The above quantity is clearly nonnegative and moreover $O(\vec{f}) \in[0,1]$. It is equal to unity if $A_{i}=\mathcal{M}_{i}$. After the maximization of the overlap the new minimization process is started as described above. The procedure has been continued until the value of $\left|\vec{A}^{(n)}-\vec{A}^{(n-1)}\right|<\epsilon$, with an admissible tolerance $\epsilon>0$. This strategy will be called as "self-consistent" Maximum Entropy Method.

\section{Structure of the library}

\subsection{General overview}

LINPRO is an object-oriented library for solving linear inverse problems written in $\mathrm{C}++$. As an optimization engine it uses $\mathrm{OPT}++2.4$ library [8]. The aim of the LINPRO library is to collect in one place various algorithms for solving inverse problems and provide unified and user friendly programming interface to all of them. The library can be used to solve the problem with an arbitrary kernel defined by the user. For the MEM the package provides an interface for defining the arbitrary default model as well as a class of default models parametrized by a set of parameters. 


\subsection{Installation and technical documentation}

To install the library unpack tarball and follow instructions contained in INSTALL file. The distribution contains also folder doc where the technical and the API documentation is located. The documentation is generated in the HTML format, the master file is index.html. The user will find the codes which solve the artificial problem (as presented below), in the attached folder: examples.

\subsection{Inverse problem solvers}

Within LINPRO library algorithms for solving the inverse problem are called solvers, represented by InverseProblemSolver class. Fig. 1 presents the inheritance diagram of available solvers.

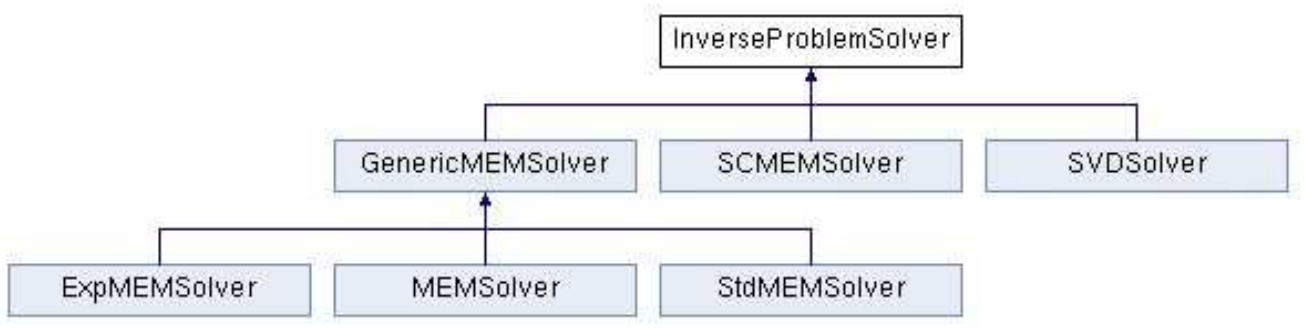

Figure 1: (Color online) Inheritance diagram for InverseProblemSolver.

The solvers represents algorithms:

- StdMEMSolver - standard implementation of the Maximum Entropy Method. To minimize the likelihood function the algorithm reduces the minimization problem to the iterative process of consecutive least square problems, as described in section 4.2 .

- MEMSolver - implementation of the Maximum Entropy Method. It minimizes the likelihood function using the nonlinear interior-point method.

- ExpMEMSolver - implementation of the Maximum Entropy Method, where object $A(x)$ is parametrized by the formula $A(x)=\mathcal{M}(x) \exp f(x)$, where $\mathcal{M}(x)$ is a model function and $f(x)$ is determined by the solver. Such a substitution is often used to eliminate the term $\log A(x) / \mathcal{M}(x)$, 
which in specific situations is a source of optimizer instabilities (for example such instabilities can occur if a chosen model $\mathcal{M}(x)$ is very close to zero for some values of $x$, and due to finite precision is treated as zero). To minimize the likelihood function the nonlinear interior-point method is used. Since this solver is much slower than standard solvers it should be used in cases when StdMEMSolver and MEMSolver do not converge properly.

- SVDSolver - implementation of the SVD method.

- SCMEMSolver - implementation of the self-consistent engine for Maximum Entropy Method. It works with each solver belonging to GenericMEMSolver branch. The scheme of the solver algorithm presents Fig. 2 ,

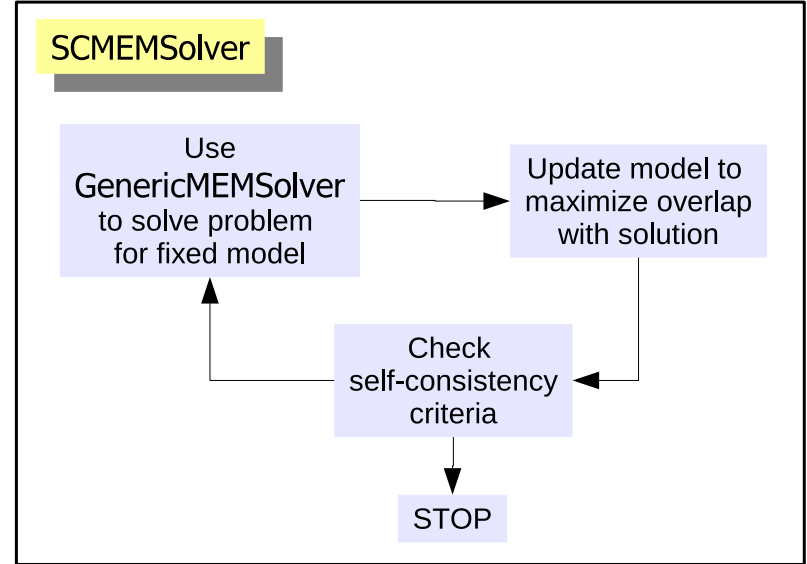

Figure 2: (Color online) The algorithm scheme for SCMEMSolver.

\section{Example of physical application}

\subsection{Problem formulation}

As an example the package has been applied to extract the spectral weight function $A(x)$ through the analytic continuation of the imaginary time propagator $G(y)$ :

$$
G(y)=-\frac{1}{2 \pi} \int_{-\infty}^{\infty} d x A(x) \frac{\exp (-x y)}{1+\exp (-x \beta)}
$$


By definition, $A(x)$ fulfills the following constraints:

$$
\begin{aligned}
& A(x) \geq 0, \quad \int_{-\infty}^{+\infty} \frac{d x}{2 \pi} A(x)=1, \\
& \int_{-\infty}^{+\infty} \frac{d x}{2 \pi} A(x) \frac{1}{1+\exp (x \beta)}=-G(\beta) .
\end{aligned}
$$

This problem is frequently encountered in Quantum Monte Carlo simulations, which by construction produce data affected by the statistical noise [9, 10]. In order to check the reconstruction ability of the package an artificially generated data for the imaginary time propagator has been used. The application of the package to the real physical data can be found in Refs. [10].

The artificial spectral function has been chosen in the form:

$$
A(x)=\frac{1}{2} N(x ;-1.5,0.5)+\frac{1}{2} N(x ; 2.0,0.7),
$$

where $N(x ; \mu, \sigma)$ is the normal distribution function with the mean $\mu$ and the standard deviation $\sigma$. Subsequently the values of the imaginary time propagator has been generated using the relation (36) for $N_{\tau}$ uniformly spaced data points in the interval $[0, \beta=10]$.

\subsection{Data without noise}

As a first step the quality of reconstruction of the object as a function of the number of data points $N_{\tau}$ has been tested. It was found that in order to reproduce the original object with a satisfactory accuracy, one has to use $N_{\tau} \geqslant 20$ data points; see Fig. 3. Further increase in the number of data points does not improve significantly the conformity between the solution and the object. It is related to the fact that the dimension of the subspace $\mathcal{A}_{M}$ increases linearly with an increase in the number of data points up to $N_{\tau}=20$ and then it saturates (for the presented example the dimension of $\mathcal{A}_{M}$-space is 20 for $N_{\tau}=20$ and 25 for $\left.N_{\tau}=100\right)$. Note also that the projected solution produced by the SVD method provides a very good approximation of the "true" object.

\subsection{Data with noise}

In order to test the ability of reconstruction in the presence of noise each value $G_{i}$ has been perturbed by the Gaussian noise of zero mean value and 


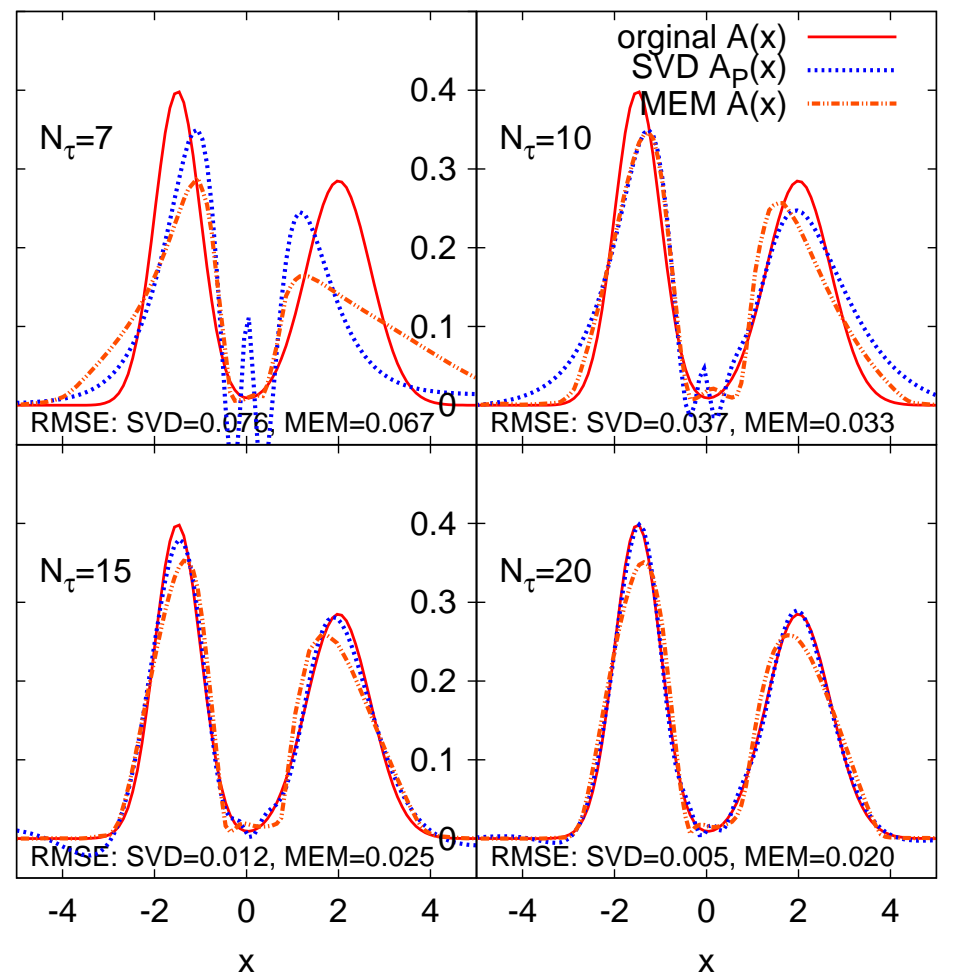

Figure 3: (Color online) The reconstruction of the artificial object function $A(x)$ by the SVD and MEM methods. The reconstruction is performed using $N_{\tau}$ uniformly spaced data points $G_{i}$ in the interval $[0, \beta=10]$ (noiseless data). A very good agreement between the normal solution and the original object is achieved if $N_{\tau} \geqslant 20$. The root mean square error (RMSE) for both methods is displayed at the bottom of the figure.

the standard deviation equal to $1 \%$ of $G_{i}$. In the case of an SVD method the object has been reconstructed using Eq. (17) for various cut-off parameters $\lambda_{i} / \lambda_{1}$ ( $\lambda_{1}$ is the highest singular value); see Fig. 4. Note that solutions with a cut-off parameter bigger than the relative error 0.01 do not guarantee the reproduction of the imaginary time correlator within its error bars. It is clearly seen that with a decreasing value of the cut-off parameter the quality of reconstruction increases, and the "optimal" cut-off parameter is $\lambda_{i} / \lambda_{1} \approx 0.01$. Further decrease in the cut-off leads to the inclusion of an unjustified structure (strong fluctuations) into the shape of the reconstructed object.

In the case of MEM the quality of reconstruction is a function of $\alpha$ pa- 


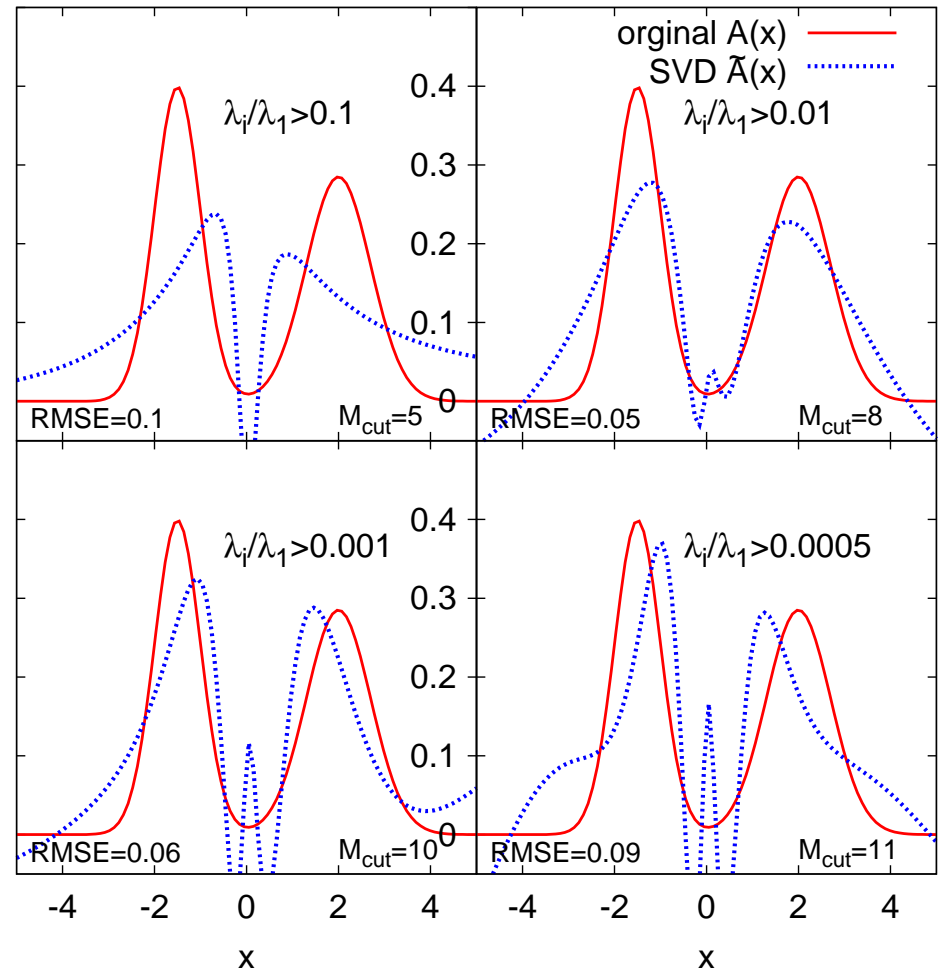

Figure 4: (Color online) Reconstruction of the artificial object function $A(x)$ by TSVD method. Reconstruction is performed using $N_{\tau}=25$ uniformly spaced data points $G_{i}$ in the range $[0, \beta=10]$, perturbed by Gaussian noise $\Delta G_{i}=\mathcal{N}\left(0, G_{i} / 100\right)$. In the reconstruction procedure only those terms were included for which $\lambda_{i} / \lambda_{1}$ is larger than a given cut-off parameter. $M_{\text {cut }}$ denotes the number of the singular function included in TSVD expansion.

rameter; see Fig. 5, A class of assumed models has been chosen according to the prescription:

$$
\mathcal{M}\left(x ; c_{1}, c_{2}, \mu_{1}, \mu_{2}, \sigma_{1}, \sigma_{2}\right)=c_{1} N\left(x ; \mu_{1}, \sigma_{1}\right)+c_{2} N\left(x ; \mu_{2}, \sigma_{3}\right) .
$$

It was found that there exists a critical value of $\alpha$ parameter which separates "smooth" and "rigged" solutions. It corresponds to the value which minimize the total MEM errors as discussed in Ref. [11]. Moreover, it turns out that the "self-consistent" algorithm always converge to the same solution irrespective of initial values of parameters which define the class of models. 


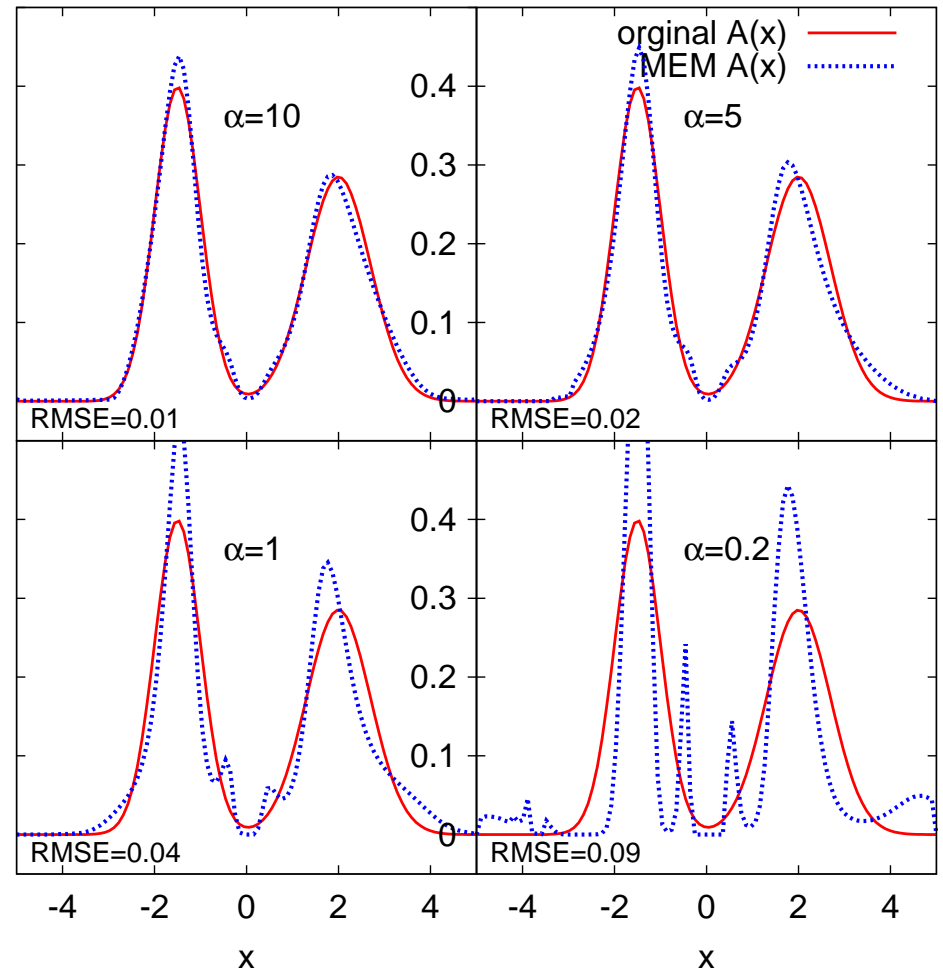

Figure 5: (Color online) Reconstruction of the artificial object function $A(x)$ by selfconsistent MEM for different values of parameter $\alpha$.

\subsection{Impact of external constraints and a priori information}

In the following the impact of the external constraints on the reconstruction quality has been tested for both MEM and SVD methods; see Fig. 6. The external constraints influence strongly the SVD method. Note also that the solution produced by an SVD method is not an accurate reconstruction of the input spectral function. It is due to the fact that the solution produced by the SVD method is a projection of the "true" spectral function onto the "visible" subspace, where the problem is well posed. The main advantage of an SVD approach is that it does not require any a priori information. Consequently the SVD solution can deliver very useful information concerning the default model or a class of default models for MEMs. In this particular test the SVD solution suggests that it is profitable to choose the default model as a combination of two Gaussians (left panel), given by Eq. (40). One can also use the SVD solution as a default model for MEMs. 
The tests presented above suggest that the maximum entropy method combined with "self-consistent" engine provides the most accurate solutions. Even if the class of the models is not correctly prepared, the self-consistent solution still well reproduces the input object. The right panel presents the case where the class of default model Gaussian functions $N(x ; \mu, \sigma)$ was used.

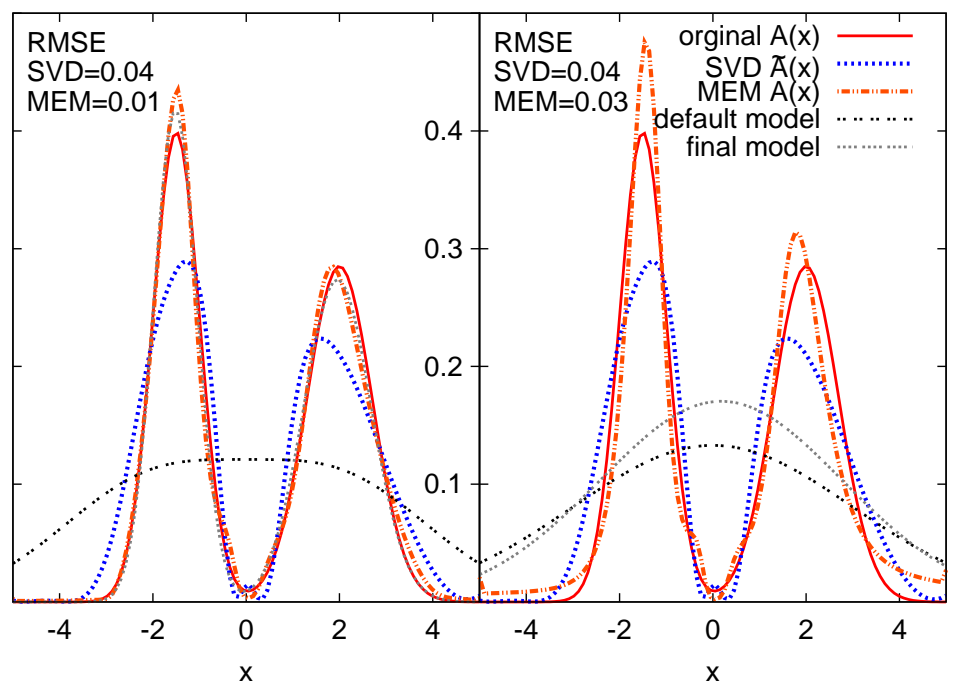

Figure 6: (Color online) The reconstruction ability of the spectral function for the full problem (data with noise + external constraints) of the SVD and MEM methods. The left panel shows the solution of the self-consistent MEM with a combination of two Gaussians functions as a default model class. The right panel shows the solution of the self-consistent MEM with Gaussian functions as a default model class.

Therefore the best methodology of producing the solution is suggested to be the following:

1. Create an SVD solution and apply it to construct the class of default models $\mathcal{M}(x ; \vec{f})$;

2. Use the "self-consistent" MEM with constructed class of models $\mathcal{M}(x ; \vec{f})$ to produce final solution.

\subsection{Resolution limit}

In the case of physical applications where the object is associated with the spectral weight function, an extremely important question needs to be 

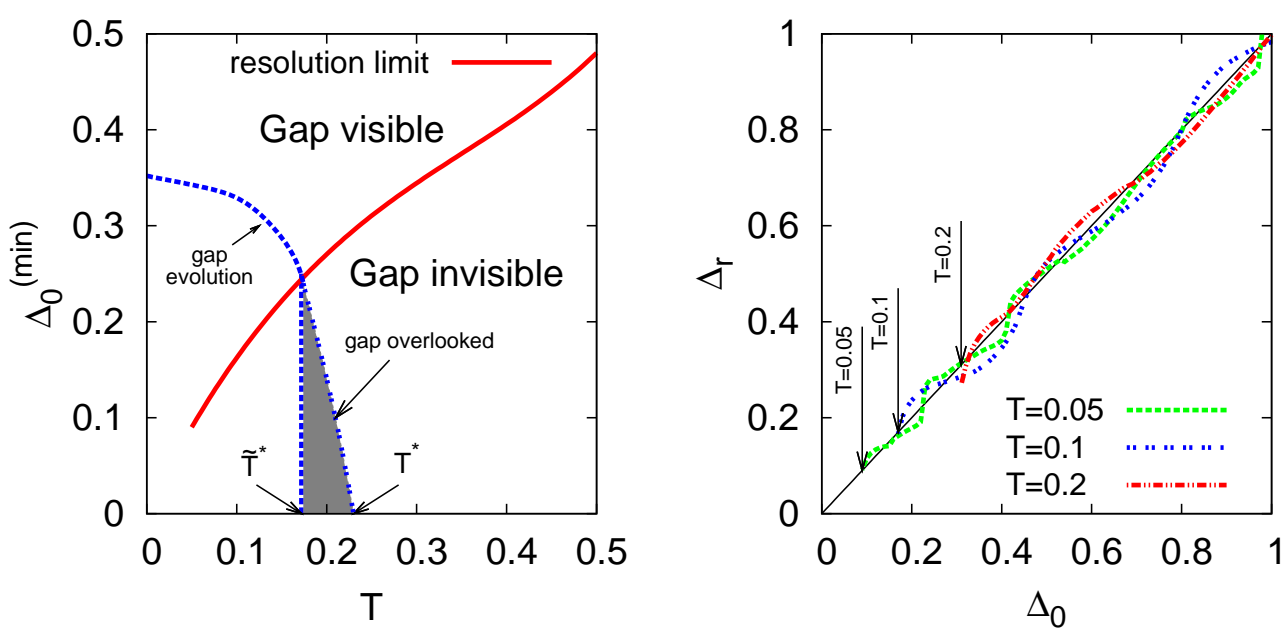

Figure 7: (Color online) Left panel: the resolution limit as a function of temperature $T=1 / \beta$ (red solid line). Above the limit it is possible to reconstruct the gap (defined as a distance between two peaks). The sketch of a typical evolution of the physical gap (dotted blue line) is also plotted. The gap can be properly reconstructed up to $\tilde{T}^{*}$ temperature. $T^{*}$ denotes the temperature for which the gap vanishes, assuming that the reconstruction provide an exact object. Right panel: the value of the gap $\Delta_{\mathrm{r}}$ reconstructed by the SVD method versus the true value $\Delta_{0}$. The support function is $S(x,-2,2)$. Arrows indicate the minimal value of the gap (resolution limit), where the bimodal structure of the reconstructed spectral function appears.

answered: is the spectral function unimodal or bimodal? It is well known that the distinct peaks of a bimodal spectral function may be overlooked during the reconstruction process if the distance between peaks is smaller than some critical value, which defines the resolution limit.

To quantitatively estimate the resolution limit, the artificial object function consisting of two delta functions separated by $2 \Delta_{0}$ distance have been considered. Namely, $A(x)=\delta\left(x+\Delta_{0}\right)+\delta\left(x-\Delta_{0}\right)$. For this function the imaginary time propagator has been generated for $N_{\tau}=25$ uniformly distributed data points in the interval $[0, \beta]$, where now $\beta$ is treated as a parameter (in this case the inverse of $\beta$ has the physical meaning of temperature). The resolution limit $\Delta_{0}^{(\min )}$ is defined as a minimal value of $\Delta_{0}$ for which the bimodal structure of the object can still be reconstructed and in general is a function of $\beta$.

Results of the presented tests are shown in Fig. 7]. For both methods 
(SVD and MEM) the existence of the finite resolution limit has been found. It increases with a decreasing temperature $T=1 / \beta$ (left panel). Within the presented approach it is possible to reconstruct the gap (defined as a distance between two peaks) only for the temperatures for which it is larger than the reconstruction limit.

Let us consider a process of reconstructing the physical gap, which is a decreasing function of temperature and eventually vanishes at some temperature $T^{*}$. At a certain temperature $\tilde{T}^{*}<T^{*}$ the gap becomes comparable with the reconstruction limit. Up to this temperature the reconstructed value of the gap $\Delta_{\mathrm{r}}$ agrees very well with the true value $\Delta_{0}$ (right panel). At the temperature $\tilde{T}^{*}$ the value $\Delta_{\mathrm{r}}$ drops to zero. It means that the methods provide in practice a lower bound for the temperature at which the true gap vanishes.

\section{Conclusions}

Library LINPRO for solving arbitrary linear inverse problems with external constraints has been presented. The library uses the Maximum Entropy Method and the SVD methods. An object-oriented implementation ensures that the package acquires a unified and user friendly interface. As an example we have applied the new package to solve the typical problem of computational physics: analytic continuation of imaginary time propagator to real frequencies.

\section{Acknowledgments}

The support from the Polish Ministry of Science under contract N N202 128439 and from the DOE under grants DE-FG02-97ER41014 and DE-FC0207ER41457 is acknowledged. One of the authors (G.W.) acknowledges the Polish Ministry of Science for the support within the program "Mobility Plus - I edition" under contract No. 628/MOB/2011/0. This work has also been partially supported by COMPSTAR, an ESF Research Networking Programme. Calculations were performed at the Interdisciplinary Centre for Mathematical and Computational Modelling (ICM) at Warsaw University.

\section{References}

[1] M. Bertero, C. de Mol, E.R. Pike, Inverse Problems 1, 301 (1985). 
[2] C.E. Creffield, et al., Phys. Rev. Lett. 75, 517 (1995).

[3] M. Bertero, C. de Mol, E.R. Pike, Inverse Problems 4, 573 (1988).

[4] G.D. de Villiers, B. McNally, E.R. Pike, Inverse Problems 15, 615 (1999).

[5] M. Bertero, P. Boccacci and E.R. Pike, Proc. R. Soc. London A 383, 15 (1982); Proc. R. Soc. London A 393, 51 (1984); M. Bertero, P. Branzi and E.R. Pike, Proc. R. Soc. London A 398, 23 (1985).

[6] E.T. Jaynes, in The Maximum Entropy Formalism, edited by R.D. Levine and M. Tribus, (MIT Press, Cambridge, 1978), pp. 15-118; R.N. Silver, D.S. Sivia, J.E. Gubernatis, Phys. Rev. B41, 2380 (1990); S.R. White, Phys. Rev. B44, 4670 (1991).

[7] S.E. Koonin et al., Phys. Rep. 278, 1 (1997).

[8] OPT++: An Object-Oriented Nonlinear Optimization Library, https://software.sandia.gov/opt++/

[9] J. E. Gubernatis, et al., Phys. Rev. B 44, 6011 (1991); M. Jarrel, J.E. Gubernatis, Phys. Rep. 269, 133 (1996); O. Gunnarsson, M. W. Haverkort, and G. Sangiovanni, Phys. Rev. B 82, 165125 (2010);

[10] P. Magierski, et al., Phys. Rev. Lett. 103, 210403 (2009); G. Wlazłowski, P. Magierski, Phys. Rev. C 83, 012801(R) (2011); P. Magierski, G. Wlazłowski, A. Bulgac, Phys. Rev. Lett. 107, 145304 (2011).

[11] O. Gunnarsson, M. W. Haverkort, and G. Sangiovanni, Phys. Rev. B 81, 155107 (2010). 